\title{
LA LÓGICA Y SU FILOSOFÍA A COMIENZOS DEL SIGLO XIX: J. D. GERGONNE
}

Hubo un momento, a comienzos del siglo xIx, en que los manuscritos lógicos de Leibniz estaban guardados — demasiado guardados-, el dictum kantiano sobre la perfección e imperfectibilidad de la lógica aristotélica había sido pronunciado - y dominaba-, y Boole estaba aún lo bastante lejano como para permitir cualquier predicción sobre el sentido de la transformación que más tarde realizarian él y sus sucesores.

Hoy sabemos cuáles fueron los momentos nodales del desarrollo posterior durante dicho siglo; sabemos en qué forma De Morgan y Peirce construyeron la lógica de relaciones, cómo Schröeder sistematizó el conjunto de la lógica algebraica de su momento, cómo Frege -en su Begriffschrift y en el resto de su obra - nos dio no sólo una, sino varias ideas fundantes decisivas, cómo Peano relacionó ciertos descubrimientos lógicos con las bases de la aritmética.

Después de la bien conocida serie de destellos _-de los que hemos recordado sólo algunos - encontramos un desarrollo continuo que comenzó con Principia Mathematica; se trata allí de una ciencia que está ya establecida y no los momentos separados de un temprano esfuerzo constitutivo. Desde los Principia, la lógica es no sólo una ocupación profesional sino, más que ello, una disciplina con resultados significativos; no sólo una herramienta posible, sino una en integración concreta con el corpus del conocimiento científico, como lo es la masa de lógica incluida en las numerosas revistas especializadas hoy.

Cuando las mismas palabras de Kant eran oídas y seguidas - y lo fueron por un lapso demasiado extenso, felizmente casi sólo por historiadores y filósofos (recordemos la influencia nefasta de la Geschichte der Logik im Abendlande de Prantl)-, el proceso de transformación de la lógica ya había comenzado. La "perfección" de la lógica anterior no era tal y su limitación era obvia aun para aquellos lógicos ocupados con aspectos polifacéticos del silogismo. Podemos decir que la lógica se estableció como la ciencia que conocemos hoy desde el momento en que los detalles del silogismo dejaron de ser el tema cotidiano de los lógicos. Y llevó mucho tiempo para que se alcanzara esa etapa. De cualquier modo, consideraré aqui a José-Díez Gergonne como uno de los lógicos cuyo tema principal en lógica era el silogismo, pero también de los que hicieron pronto que el dictum kantiano fuera obsoleto, que comenzaron a realizar el ideal leibniziano y que trabajaron de un modo casi booleano.

Es sabido que, dentro de la tradición lebniziana, Lambert, Ploucquet, Holland, los Bernouilli y Castillon trataron de construir un cálculo de la lógica basado en una consideración intensional de la proposición; por esa misma razón, sus esfuerzos no tuvieron éxito ni fueron muy positivos para 
el desarrollo ulterior de la lógica tal como la conocemos hoy. Boole tuvo que comenzar de nuevo en su Mathematical Analysis of Logic. Bochenski ${ }^{1}$ considera a aquellos lógicos junto con Gergonne, porque ninguno de ellos creó una escuela que fuera persistente; sin embargo, parecería con todo que el caso de Gergonne es muy diferente que los demás. Veremos más adelante las razones de ello.

2.I Examinemos el caso de Gergonne. En otras ocasiones he considerado en detalle su algoritmo lógico. ${ }^{2}$ No enfocaré el presente trabajo sobre su cálculo lógico porque otros aspectos me interesan más aquí para situar a Gergonne en el desarrollo de la lógica en el siglo xIx. Sin embargo, indicaré sus líneas principales remitiéndome para ello a los trabajos citados. ${ }^{3}$

2.1.1 La base del cálculo ${ }^{4}$ es la consideración de las extensiones de las ideas a través del uso de sus representaciones gráficas como figuras cerradas, convexas, planas (círculos). En primer lugar, el uso de las extensiones como base para un cálculo no era la costumbre; como dijimos antes, los esfuerzos por construir cálculos con una base intensionista se vieron condenados al fracaso en manos de los sucesores de Leibniz, en razón de las dificultades para manejar las intensiones simbólicamente. El uso sistemático de las extensiones tuvo, para Gergonne - como para Boole más tarde-, un efecto fructífero.

En segundo lugar, Gergonne usó consistentemente, como dijimos, las representaciones gráficas a través de su cálculo. Ahora bien, la mayor parte de los lógicos que, antes y después de ell, presentaron gráficos para su uso lógico, se limitaron a la mera representación sin usarla profusamente, como lo hizo Gergonne, en el desarrollo de los algoritmos; sólo muy posteriormente Venn y Ladd-Franklin son excepciones en la serie de los seguidores lógicos de Euler.

Pero el principal artificio que permite extraer consecuencias de los otros - es decir, del cálculo y la representación gráfica - es el uso de tablas lógicas. El algoritmo de Gergonne se constituye a través de una manipulación de tablas que combinan las extensiones con la representación gráfica de las relaciones entre ideas.

El cálculo aludido, aplicado a los elementos del silogismo, produjo resultados que fueron sólo parcialmente coincidentes con los de las formulaciones tradicionales, lo cual permitió la introducción de rectificaciones parciales en ellos. El cálculo de Gergonne merece realmente ese nombre; es un procedimiento que produce resultados sin la intervención de elementos ajenos al cálculo. El llamado cálculo lógico establecido por Castillon ${ }^{5}$ exigía muy a menudo correcciones arbitrarias, provenientes de las ya existentes reglas tradicionales y por tanto externas al "cálculo" mismo.

1 A History of Formal Logic.

2 "Dos precursores de la lógica algorítmica".

3 Fundamentalmente Gergonne' a teoria definicjii; Venn, J., Symbolic Logic, último capítulo; Vacca, G., "Sui precursori della logica matematica"; Lewis, C. I., A Survey of Symbolic Logic; Enriques F., Para la historia de la lógica.

4 "Essai de Dialectique Rationelle".

5 "Mémoire sur un nouvel algorithme logique". 
A partir de una base ampliada -cinco relaciones básicas entre extensiones- Gergonne desarrolló un procedimiento formal de calcular. Aunque se originaba en la consideración del silogismo, no estaba en principio limitado a él. Faris lo ha mostrado con claridad. El algoritmo ya posee, en ese sentido, algunas de las características más destacadas del desarrollo posterior de la lógica: una simbolización adecuada, el uso de las extensiones, la utilización amplia de representaciones gráficas, la construcción de tablas lógicas, un cálculo en gran medida abstracto, la posibilidad de una interpretación consistente de los resultados en relación con la base asumida... Pero eso no es todo. Gergonne observó que las veinticuatro formas válidas de silogismo que obtiene a través del cálculo no son primitivas; no sólo cinco de ellas son supertluas (con lo cual se retornaría a las clásicas diecinueve en la mayor parte de las teorías corrientes), sino que también lo son muchas otras.

Gergonne deriva de su cálculo teoremas concernientes a las relaciones entre proposiciones y enuncia un principio de reducción de formas válidas que es bien conocido: un silogismo es válido cuando la contradicción de su conclusión junto con una de las premisas forma otro silogismo válido cuya conclusión es la contradictoria de la otra premisa, principio que fue usado luego para construir el método llamado del antilogismo. Todas las formas válidas se reducen primeramente a dos y luego a sólo una.

2.1.2 Lo que hemos dicho muestra que la contribución de Gergonne a la lógica formal no implica por cierto una revolución; su Dialectique Rationelle continúa apegada tanto a los resultados y a algunas interpretaciones básicas de la lógica tradicional, como a su tema, el silogismo. Tiene, con todo, ciertas características que la distinguen netamente de un manual corriente de lógica escolástica o aun de una contribución erudita a ésta. Es interesante señalar que, mientras que otras tentativas de simbolización trataron de cuantificar la lógica introduciendo números, de un modo infundado y casi siempre arbitrario, Gergonne, matemático él mismo, realizó una "formalización" no-aritmética de la lógica. El simbolismo y el algoritmo tabular que desarrolla le permiten reconstruir de modo mecánico las leyes de la inferencia inmediata y del silogismo. Eso implica, al mismo tiempo, una verificaci6n parcial del algoritmo mismo y la posibilidad de afinar los resultados que la lógica tradicional debia a un análisis basado excesivamente en los lenguajes naturales, a partir de los cuales había sido construido. Aun si los resultados del cálculo sólo difieren en detalle de los obtenidos en la lógica tradicional, su manera de manejar la lógica pasó a ser completamente distinta de la dominante. $\mathrm{La}$ empresa de Gergonne mostró que una superación definitiva de la lógica clásica en el marco del silogismo no era posible. Por ello, la concepción que presidió dicha empresa constituye un antecedente muy importante de la transformación que iba a ocurrir treinta años después en otras manos.

Los símbolos que Gergonne utiliza no son los mejores para sus propósitos pero son claramente superiores a los usados mucho más tarde por Delboeuf. Cuando en 1877, treinta años después de la obra básica de Boole, Delboeuf publicó su libro, ${ }^{6}$ utilizó una simbolización y una representación

${ }_{6}^{6}$ Logique algorithmique. 
gráfica tan complicadas que sólo con gran esfuerzo obtuvo resultados que habían sido logrados con anterioridad. Recordemos además la lentitud con que han ido apareciendo estudios de las obras de Frege y de Peirce en lógica, debido en parte a las simbolizaciones y terminologías utilizadas, y a pesar de que sabemos que sus obras contienen atajos fundamentales.

El sistema de Gergonne no sólo está expresado con una notación relativamente conveniente para sus propósitos, sino que muestra asimismo coherencia y simplicidad suficientes. Prueba la suficiencia y necesidad de las propiedades que establece y enuncia con claridad las formas de razonamiento válidas en su sistema.

Debe señalarse especialmente la intercambiabilidad que Gergonne muestra entre algunos de sus principios y ciertos teoremas. Sin embargo, veremos más tarde que sus concepciones sobre este asunto no son completamente claras ni consistentes.

2.1.3 Gergonne indica en diferentes lugares, especialmente en la Dialectique Rationelle ${ }^{7}$ y en el Essai sur la théorie des définitions, ${ }^{8}$ la similitud entre los métodos que trata de aplicar en lógica y los procedimientos del álgebra:

On doit ajouter encore qu'assez souvent on peut raisonner sur les mots sans qu'il soit besoin de s'enquérir de leur signification, tout comme en algèbre on exécute des calculs, sans songer aucunement à ce que représentent les symboles sur lesquels on opère.

Aunque Gergonne no desarrolla todas las consecuencias posibles de ese hecho, su tratamiento del silogismo revela claras similitudes con los procedimientos algebraicos.

En ese sentido, Gergonne avanza un esquema de la posición booleana en cuanto a la interpretación de los símbolos. Podemos comparar la cita anterior con las siguientes palabras de Boole:

Every system of interpretation which does not affect the truth of the relations supposed, is equally admissible, and it is thus that the same process may, under one scheme of interpretation, represent the solution of a question on the properties of numbers, under another, that of a geometrical problem, and under a third, that of a problem of dynamics or optics. ${ }^{9}$

De ese modo, Gergonne produce un algoritmo similar al algebraico. EK objetivo es obtener mecánicamente los resultados sin la necesidad de razonamiento, lo que constituye, en una palabra, "le comble de la perfection des méthodes". 10

7 Pp. 206-7 y 211.

8 Pp. 11 y 13.

9 The Mathematical Analysis of Logic, p. 3.

10 "Essai de Dialectique Rationelle", p. 215 n. 
Aun si la principal característica de la nueva lógica desde mediados del siglo XIX, es su transformación en un cálculo ( $y$ en este aspecto señalamos ya el aporte de Gergonne y su importancia), el sentido profundo de la contribución de Gergonne a la lógica surge de una fuente muy diferente: la subyacente filosofía de la lógica y del lenguaje y su teoría de la definición. Son los aspectos que consideraremos.

2.2 El ensayo sobre el análisis y la síntesis en las ciencias matemáticas no cubre sólo este tema - que ha perdido buena parte de su interés para nosotros - sino que complementa la Dialectique Rationelle y adelanta en aspectos importantes su teoría de la definición. Dice: "Démontrer un théorème, c'est en général prouver par un raisonnement exact que ce théorème est une conséquence inévitable d'un ou de plusieurs autres théorèmes antérieurement admis." "11 Por otra parte, su terminología es peculiar para su época: llama teoremas a lo que posteriormente se conocerá como tesis de un sistema (las proposiciones afirmadas, sean axiomas o teoremas). $\mathrm{Y}$ afirma inmediatamente que:

...Il existe heureusement, des théorèmes dont il suffit de connaître l'énoncé pour en apercevoir la vérité. 12

Pero ya hemos visto que la Dialectique Rationelle contiene una concepción diferente sobre el status de los axiomas. En ese sentido, Gergonne no es consistente: en algunas partes afirma la intercambiabilidad de las tesis del sistema y en otras exige la evidencia y la necesidad de algunas de ellas, los axiomas. Aun durante el siglo que asistió a una transformación crucial en este sentido, encontramos inconsistencias en los escritos de los principales autores de ese cambio [recordemos que Russell en Foundation of Geometry (1897) todavía distinguía en los postulados entre "the necessary and the purely assertorical"]. Sin embargo, la terminología misma de Gergonne apunta en la dirección del cambio que la Dialectique Rationelle anuncia y que su geometría proyectiva impulsa mucho más.

Se debe subrayar que Gergonne incluye, ya en su trabajo, las definiciones entre los axiomas en el sentido de que no son dependientes sino elegidas: insiste en que las definiciones nominales, las diferencias no-"esenciales", y los significados no-intrínsecos, ${ }^{13}$ son los únicos aceptados en ciencia. Y la misma elección del punto de partida (axiomas, definiciones, hipótesis en las demostraciones) es puesta de relieve a tal grado que podemos dudar acerca de la evidencia reclamada de tanto en tanto para los axiomas.

On doit remarquer ici que, dans une science, il y à peu de proposition qui ne puisse être considerée comme une espèce de centre où viennent également concourir les conséquences d'une multitude d'autres proposi-

11 "De l'analyse et de la synthèse dans les sciences mathématiques", p. 345.

12 Ibid., p. 345.

13 Ibid., pp. $346 n$., 364 n. y 369. 
tions, dont chacune conséquemment pourrait, à son tour, être prise par point de départ dans le raisonnement qui doit établir l'autre. ${ }^{14}$

Lo que dice de este modo acerca de los teoremas intermediarios lo pone en práctica respecto a los teoremas básicos (los axiomas) en la Dialectique Rationelle. La posición central de la noción de elección en su análisis es por ello una característica reveladora.

2.3 Cuando Gergonne distingue cinco casos posibles de inclusión-exclusión de clases, señala: 1) que no existe ningún lenguaje natural en el cual las proposiciones estén construidas sobre esa base, 2) que un lenguaje que fuera construido de aquel modo sería mucho más preciso que los existentes, y 3) que su "dialéctica racional" sería muy diferente de la de aquéllos.

Por ello Gergonne entiende que la lógica necesita trascender la estructura de los lenguajes naturales sobre cuya base ha sido originalmente construida; piensa que la "formalización" (por medio de simbolización adecuada y cálculo puro) es uno de los caminos para ello; pero realiza sólo parcialmente la tarea que considera necesaria, aun si su Dialectique Rationelle intenta, como vimos, despegarse de las estructuras gramaticales por medio de un símil algebraico. Si Gergonne hubiera alcanzado tal liberación de las formas naturales del lenguaje, habria dado un paso fundamental hacia la constitución de la lógica matemática. Pero la limitación de la dialéctica racional al silogismo impide un tipo de consideración que pueda formalizar el razonamiento en un grado suficiente. Gergonne mismo reconoce que la "formalización", por lo menos en aquella etapa, sólo se aplica en sentido estricto a las ciencias exactas (opinión de cualquier modo excesivamente optimista para su época), porque éstas poseen un avanzado grado de formalización en ellas mismas.

El avance de las ciencias está condicionado entonces por una elección de su lenguaje:

Il est donc vraje de dire qu'en créant ou en perfectionnant une science on se trouve inévitablement conduit à créer ou à perfectionner une langue. ${ }^{15}$

En ayant donc l'attention... de remplacer une collection de mots par un mot unique équivalent, il arrivera que les propositions placées aux derniers limites des sciences ne seront pas plus compliquées que les propositions élémentaires desquelles elles auront été deduites. ${ }^{16}$

La definición es, por esa misma razón, la herramienta principal en la creación de un lenguaje científico y en el avance de la ciencia. Gergonne da una justificación válida aún hoy -frente a muchos ataques infundadospara el uso del lenguaje científico:

C'est donc bien à tort que l'on reproche aux savans de ne point parler

14 "De l'analyse et de la synthèse dans les sciences mathématiques, p. 347 n.

15 "Essais sur la théorie des définitions", p. 11.

16 Ibid., p. 11. 
la langue vulgaire, et d'en créer une exclusivement destinée à leur usage; c'est au fond leur reprocher de s'occuper d'autres objets que ceux dont s'occupe le vulgaire, ou d'envisager les objets sous d'autres rapports. Ce n'est pas volontairement, c'est tout-à-fait par contrainte qu'ils créent des mots nouveaux à mesure qu'ils pénètrent plus avant dans leurs recherches. ${ }^{17}$

Pero tampoco exagera la función del lenguaje en ciencia; le atribuye sólo un mérito derivado en el desarrollo científico:

Loin donc que l'extrême rigueur que personne n'a jamais songé à contester aux sciences mathématiques ait sa cause dans la perfection du langage de ces sciences; il y a beaucoup plus d'apparence que c'est au contraire à cause de l'extrême simplicité des objets auxquels ces sciences sont relatives, qu'on a pu parvenir à donner tant de precision à la langue qui en exprime la nature et les propriétés. ...C'est que le progrès de la science n'est pas tant l'éffet que la cause du perfectionnement de la langue qui lui est prope. ${ }^{18}$

2.4 Los tres trabajos filosóficos que estamos utilizando ${ }^{19}$ aparecieron en los Annales de Mathématiques Pures et Appliquées que Gergonne publicó en Nismes durante la primera mitad del siglo xIx. Era uno de los periódicos cientfficos importantes de su época - y la primera revista estrictamente matemática que se haya publicado, en un momento en que el amplio movimiento de revistas científicas se consolidó y apuntó hacia su expansión explosiva actual. La mayor parte de los trabajos que aparecieron en los Annales enfocaban el desarrollo de las ciencias matemáticas (no sin sus curiosidades y aplicaciones: algunos dedicados al estudio matemático de las votaciones parlamentarias, de las votaciones en general y de las realizadas en el ejército francés revolucionario para elegir sus oficiales; algunos al estudio de fenómenos económicos, sin contar con las aplicaciones físicas; adelantan en su espiritu ciertas preocupaciones y tratamientos mucho más recientes).

Como bien se sabe, las matemáticas del siglo xxx participaron en el desarrollo de la lógica desde su comienzo; en algunas de sus ramas son, por sí mismas, lógica aplicada avant la lettre, y en muchos aspectos plantearon las principales cuestiones que la nueva lógica trató de responder; más aún, la nueva lógica nació para responderlas. Recordaré aquí una de esas ramas fértiles: la geometría proyectiva. Los Annales son el testigo de su florecimiento. No sólo contienen la larga polémica entre Poncelet y Gergonne sobre la precedencia de tales descubrimientos básicos como el principio de la dualidad, sino que también nos traen varios trabajos fundamentales sobre lo que luego sería la geometría no métrica. En sus Considérations philosophiques sur les

17 "Essai sur la théorie des définitions", p. 9.

18 "De l'analyse et de la synthèse dans les sciences mathématiques", pp. 360-1, $361 \mathrm{n}$.

19 "Essai de dialectique rationelle", "De l'analyse et de la synthèse dans les sciences mathématiques" y "Essai sur la théorie des définitions". 
éléments de la science de l'étendue, Gergonne reconoce ya la importancia de una geometría que intercambia los términos primitivos de su sistema; de una geometría que, como bien lo sabemos hoy, apunta hacia la geometría abstracta; una geometría que contiene en semilla la formalización lógica. Gergonne no sólo acusa a Poncelet de llamar romántica a una geometría de cuatro dimensiones, sino que también explica con cuidado algunas de sus ideas generales concernientes a la geometría proyectiva. Gergonne atribuye mayor importancia a la reestructuración de la geometría con sentido de economía que a descubrir nuevos teoremas (aunque descubre algunos importantes); según él, el método de dualidad permite un avance mayor que la pesada dedicación a detalles. Los nuevos métodos permitirán así "una cosecha más rica de teoremas".

Desde nuestro actual punto de vista, Gergonne -en su cálculo lógico, en sus descubrimientos en geometría proyectiva, en su análisis del lenguaje científico y de la definición, y en sus consideraciones teóricas respecto a esos temas- apunta sobre todo hacia la formalización lógica, avanza el bosquejo de desarrollos ulteriores $y$, en algunos casos, comienza a dibujar el, para nosotros conocido, paisaje.

3.1 Según Gergonne las definiciones crean el lenguaje científico; sin su ayuda el lenguaje natural se complicaría a tal punto que la ciencia sería una empresa muy dificultosa. Por ello la prescindibilidad teórica de la definición está de algún modo balanceada por su ventaja psicoeconómica en el lenguaje. Este hecho está a la vez en el origen de las concepciones equivocadas de la definición (que afirman su enraizamiento objetivo en las cosas) y en el origen de la necesidad de una teoria adecuada de la definición.

3.2 La fuente de la definición es también para Gergonne una convención; una elección como la concerniente a la base general de la demostración, porque la definición no es sino otra herramienta de ese proceso. Los signos no portan significados por ellos mismos, sino por el propio acto de convención; la elección determina el uso futuro de los signos y en ese respecto acota el nuevo sentido. Esta determinación se refiere muy especialmente a la sustitución de un conjunto dado de signos por otro nuevo.

Ainsi, faire une définition, c'est proprement et uniquement annoncer que l'on convient d'exprimer à l'avenir, par un mot unique, choisi arbitrairement, une collection d'idées que, sans le secours de ce mot, on serait obligé d'exprimer par le moyen de plusieurs autres, et conséquemment d'ne manière moins brève. ${ }^{20}$

La définition ne fait donc autre choise qu'établir une identité de sens entre deux expressions d'une même collection d'idées dont la plus simple est nouvelle et arbitraire; tandis que l'autre, plus composée, est énoncée en mots dont le sens se trouve déjà fixé, soit par l'usage, soit par une convention antérieure. ${ }^{21}$

20 "Essai sur la thćorie des définitions", p. 13.

21 Ibid. 
Aun si Gergonne introduce la palabra 'sentido' se refiere inequívocamente al uso de signos; es decir, que enuncia una regla de sustitución del definiens por el definiendum. Por esta razón no parece adecuado cuando Kaminsky dice que "Gergonne llamó definición, en el sentido más amplio de esta palabra, a un enunciado que define (o que permite acercarse intuitivamente) a la denotación del nombre".22 Aun si Gergonne no utiliza en el trabajo el lenguaje más adecuado para sus propósitos, sin embargo alcanza su objetivo. $\mathrm{Y}$ para Gergonne el propósito de la definición no es tampoco "dar una característica sucinta de la naturaleza del objeto" como Kaminski sostiene. En el sentido verdaderamente más amplio Gergonne usa la definición como una limitación de una ambigüedad previa. Definir, para Gergonne, es limitar una indeterminación; es una reducción a lo que está ya limitado en nuestro conocimiento. Esto, como veremos, es especialmente relevante para su noción de definición implicita. Las reglas que Gergonne da para la definición dependen de esta concepción más amplia. Cada definición debe contener sólo un definiendum, y un definiens suficiente como para eliminar la preexistente ambigüedad. Las dos reglas son entonces las dos caras del mismo proceso de limitación. Gergonne nos dice que si el definiens es excesivo o si tenemos dos presuntas definiciones del mismo signo, perdemos el carácter de "arbitrariedad lícita" que posee la definición. En ambos casos tenemos en realidad una definición estricta y además un teorema (él usa, como vimos, esta expresión), que debería ser reducido o probado con respecto a la definición estricta y a los axiomas disponibles. Como vemos, dentro de sus limitaciones, la concepción de Gergonne de los sistemas deductivos ciertamente se adelanta a sú época.

3.3 Gergonne considera también otras concepciones acerca de la definición. Su bêle noire es Condillac, cuyas numerosas contradicciones señala. Pero no insistiremos en este punto. Más interesante es su observación relativa a la definición por género y diferencia. Nos dice que ese tipo de definición es adecuado sólo en un lenguaje completamente construido y presupone una clasificación terminada. Como dijimos, la definición para Gergonne es un procedimiento de limitación y no un "conocimiento completo", y sólo las ciencias exactas en su grado más avanzado de desarrollo admiten tal tipo de definición. Por esta razón, en general - fuera de este caso- sólo aparece como un artificio pedagógico y no como una herramienta en la construcción de la ciencia.

Gergonne recuerda el modo en que los "metafísicos" utilizan la definición como método de "resolver" cuestiones por medio de lo que hoy llamaríamos una analitización. La definición realmente actúa como un determinador del contexto dentro del cual la respuesta a ciertas cuestiones se hace analítica. Mediante la definición algunas cuestiones metafísicas simplemente cambian su marco de referencia.

En varios lugares Gergonne especifica qué clase de palabras son necesarias en el discurso científico (recordemos el lugar importante y distintivo que da

22 Gergonne' a teoria definicjii, resumen. 
a los términos relacionales). ${ }^{23} \mathrm{Y}$ al mismo tiempo reivindica el nominalismo ${ }^{24}$ al rechazar los significados intrínsecos y las diferencias "esenciales" tal como aparecen en las definiciones de cosas.

Nous rejetons tout à fait l'emploi des definitions de choses... il parait que cette dernière sorte de définition ne diffère uniquement de la première [nominal] que en ce que lui que l'énoncé ne prétend pas fixer le sens du mot défini; mais que, prenant ce mot suivant l'aception générale, il prétend simplement expliquer quelle est cette acception. ${ }^{25}$

De tal modo las llamadas definiciones de cosas no definen, sino que explican el uso general de las palabras y no determinan el uso de ellas; presuponen que ciertas palabras son utilizadas por todos del mismo modo; hacen prescindible en esos casos - no tan frecuentes- la definición regular, y son verdaderas o falsas en su descripción del uso natural.

Finalmente Gergonne rechaza la afirmación pascaliana de que toda idea debería ser definida y de que la imposibilidad de definir todas las palabras implica una imperfección radical de las matemáticas y del intelecto humano.

Gergonne piensa que las ideas simples son conocidas por experiencia y abstracción pero la teoría del conocimiento implícita en esto no es ahora nuestro tema. Lo que deberiamos recordar al respecto es el hecho de que hay palabras propiamente indefinidas en nuestro lenguaje, así sea cientifico.

3.4 El aprendizaje contextual del vocabulario sugiere otra clase de definición que las hasta ahora consideradas. Sin embargo, no agudizaremos las diferencias que presenta con ellas.

On conçoit fort bien, en effet, que, si une phrase contient un seul mot dont la signification nous soit inconnue, l'énoncé de cette phrase pourra souvent suffire pour nous en révéler la valeur. ${ }^{26}$

"La diagonal de un cuadrilátero lo divide en dos triángulos" es una oración que define implicitamente - la expresión es de Gergonne- a la diagonal, dando por ya definidos los otros elementos geométricos (cuadrilátero y triángulo), y por cierto el uso de términos relacionales lógicos y geométricos (cosa que Gergonne no dice). Es sólo otra forma de considerar una definición implícita, en palabras suyas:

la même différence qui existe entre les équations résolues et les équations non résolues. ${ }^{27}$

Presentando la similitud con el caso de dos ecuaciones con dos incógnitas

23 "Essai sur la théorie des définitions", p. 7.

24 Ibid., p. 6.

25 Ibid., p. 27.

26 Ibid., p. 22.

27 Ibid., p. 23. 
que determinan los valores que las satisfacen, Gergonne generaliza aún más la noción de definición implícita: $n$ frases determinan $n$ palabras dado por conocidas el resto de las palabras. Pero se trata de dos pasos muy diferentes: la noción de definición implícita en una oración, y su funcionamiento por medio de dos o más oraciones. Aun con esta generalización Gergonne exige la eliminabilidad del definiendum.

Mais il y a ici à executer une sorte d'élimination qui peut devenir d'autant plus pénible que le nombre des mots dont il s'agit est lui même plus considerable. ${ }^{28}$

La condición de eliminabilidad es entonces, por lo menos, un requisito deseado aun para el nuevo tipo de definiciones.

Vale la pena recordar una de las condiciones que Gergonne habia establecido para las definiciones explícitas: si aparece más de un término nuevo la definición no es posible: si ninguno aparece, dice Gergonne, la proposición es sólo un candidato a teorema. Requisitos similares significan que la definición implícita será válida solamente en el caso que determine las palabras definidas (dejando de lado por ahora las cuestiones más recientes relativas al isomorfismo de los modelos que satisfarían).

Ambos requisitos -eliminabilidad y relación entre el número de términos definidos y el número de oraciones - muestran que Gergonne pensaba las definiciones implícitas en un sentido más estricto del que fueron entendidas más tarde. Aparte hay que señalar que en ningún lugar del trabajo -y especificamente en ningún lugar de la página 23 de su Théorie des définitions - aplica su nueva noción a la base axiomática de los sistemas. Podria pensarse que es sólo una cuestión de los ejemplos elegidos, pero en cualquier caso los ejemplos son siempre de elementos definidos y no de "los elementos indefinidos que son definidos implícitamente por medio del conjunto de axiomas", dictum que se transformó luego en un lugar común. De cualquier modo, Gergonne no usa esa expresión ni ninguna equivalente.

4.1 Dos historias de la lógica serias y bien difundidas, aparte de muchos trabajos que se refieren al tema, repiten una y otra ve ${ }^{2 \theta}$ que J. D. Gergonne introdujo la noción de definición implícita por medio de sistemas de axiomas. Si tal hubiera sido el caso, la noción habría pasado por cuatro diferentes

28 "Essai sur la théorie des définitions", p. 23.

20 "Sometimes it is said that the axiom formulae of a system together amount to an implicit definition of the geometrical signs which they contain. This way of speaking, which was introduced by J. D. Gergonne as early as 1818 ("Essai sur la theorie des définitions", Annales de Mathématiques, v. 9,1818 , pp. 1-35, especially p. 23), "is useful but can lead to misunderstandings", Kneale, William and Kneale, Martha, The Development of Logic, p. 385); "according to the analysis of J. D. Gergonne (187I-1859) (Enriques, Per la storia della logica, ch. 3, n. 21) the modern mathematical logicians consider the primitive concepts implicitly defined from the postulates that link them, while the other concepts succesively introduced are defined explicitly in a nominal way" (Carruccio, Ettore, Mathematics and Logic in Contemporary Thought, p. 64). 
momentos: 1) la innovación de Gergonne, 2) su uso generalizado, 3) su amplio descrédito, y 4) la reivindicación de Quine.

Es muy fácil ver claramente cuándo ocurrieron 2), 3) y 4), pero muchos historiadores de la lógica y la geometría, y quienes los repiten, insisten en atribuir la invención a Gergonne, lo cual es erróneo. Es verdad que Gergonne introdujo la noción de definición implícita en su Mémoire de $1818 .^{30}$ Su análisis de la definición es adecuado y claro para su época; su noción de la definición implícita estuvo apenas a un paso de alcanzar la referente a sistemas de axiomas aparecida con posterioridad. Pero esta diferencia aparentemente pequeña puede ser bien explicada recordando el contexto de Gergonne en la historia de la lógica y el resto de su trabajo científico.

4.2 Muy posteriormente -en las Vorlesungen über neuere Geometrie de Pasch y en el sistema de axiomas para los números naturales de Peano- la noción de definición implícita por sistemas de axiomas comenzó una notoria pero corta carrera. Ya al comienzo de nuestro siglo, las dificultades de la idea se hicieron evidentes y cayó en casi total olvido. Dos de las razones de ese descrédito las estableció claramente Cooley en su libro de texto:

Postulates resemble straightforward definitions in that both are conventional (remember that a postulate is written out as an ordinary statement while a definition is explicitly written as a convention about word usage), but the former differ from the latter in that the former ordinarily provide no rule of elimination, ${ }^{31}$

Tan pronto como comenzó a ser utilizada, la noción fue criticada y muy pronto se abandonó un dictum que había estado de moda: los axiomas definen implícitamente los términos indefinidos del sistema.

La reivindicación debida a Quine ${ }^{32}$ consiste precisamente en construir un método para convertir los axiomas en definiciones, un método de eliminación por medio de la aritmética. No insistiré en este aspecto pero es interesante recordar las vicisitudes y el ciclo histórico completo de la noción.

Enriques ${ }^{3 s}$ ha sido mal citado por historiadores de la lógica que no tenían contacto directo con los trabajos de Gergonne. De tal modo se produjo el deslizamiento referido en la interpretación, y ésta fue repetida desde entonces.

4.3 Como hemos mostrado antes, , $^{34}$ Gergonne hizo varios aportes importantes a la ciencia del siglo xxx. En su Dialectique Rationelle construyó un

30 "Essai sur la théorie des définitions".

31 Cooley, John, A Primer of Formal Logic. Pap considera otro aspecto: "Closer analysis shows, however, that such 'implicit definitions' are propositional functions containing the primitives as free predicate-variables" ("Analytic truth and implicit Definitions"). Cito a Cooley y Pap como ejemplos de una perspectiva posterior sobre las definiciones impl1citas.

'32 Quine, W. V. O., "Implicit definition sustained". Quine repite también la errónea adscripción a Gergonne.

33 Enriques, F., The Historic Development of Logic.

34 Secciones 1-3 del presente trabajo. 
interesante algoritmo para mejorar la silogística. Muestra allí la necesidad de una lógica nueva y de un método para desarrollarla. A través de los resultados de dicho algoritmo enuncia explícitamente los supuestos ocultos del razonamiento silogístico. A la vez Gergonne es probablemente ${ }^{35}$ el primero en introducir el principio de dualidad en la geometría proyectiva; de hecho se cuenta entre los primeros que desarrollaron las geometrías no métricas. En su Mémoire sobre el análisis y la síntesis establece la naturaleza de los sistemas deductivos:

Démontrer un théorème c'est en général prouver, par un raisonnement exact, que ce théorème est une conséquence inévitable d'un ou plusieurs autres théorèmes antérieurement admis. ${ }^{36}$

Por ello, el algoritmo y sus consecuencias, la idea de dualidad, sus otras contribuciones a la geometria proyectiva, su clara comprensión de los sistemas deductivos y especialmente su idea de la definición implícita colocan a Gergonne en una posición importante en la historia de la lógica científica y de la matemática de comienzos del siglo xIx. Y para ello no es necesario atribuirle más de lo que logró realmente.

4.4 Gergonne indica, como hemos mostrado, cómo una definición general trabaja tanto en el lenguaje científico como en el cotidiano y da las reglas para su uso. Vale la pena recordar que admite como básicas solamente las definiciones nominales y que critica el ver en la imposibilidad de definir cada término una imperfección de las matemáticas y del intelecto humano.

Vale la pena recordar una de las condiciones que Gergonne establece para las definiciones explícitas. Un término nuevo, y sólo uno debe aparecer en cada definición; si aparecen más, la definición no es posible; si no aparece ninguno, la proposición es un candidato a teorema y por tanto exige su prueba -la cual es en sí una observación interesante. De acuerdo con esto, cuando introduce la noción de definición implícita en paralelo con un sistema de ecuaciones, Gergonne exige que el número de términos definidos sea igual al número de definiciones. En este sentido piensa en la eliminabilidad aun de las definiciones implícitas y en su transformación en explícitas.

Recordemos, con todo, que el uso posterior de las definiciones implícitas mediante sistemas de axiomas no requirió ese equilibrio numérico y no conduce, no puede conducir, a tal tipo de eliminación. Gergonne muestra que la definición implícita de las diagonales en los cuadriláteros - que contradice además la última parte de la cita de Carruccio-, y la de las palabras en el lenguaje cotidiano, siguen su análisis de la noción. Pero en cambio Gergonne no da ninguna referencia a las definiciones implícitas de los términos primitivos mediante sistemas de axiomas, porque para las definiciones implí-

35 Ver la polémica entre Gergonne y Poncelet acerca de la prioridad en el uso de esa noción (tomó varios números de los Annales; ver también "Considerations philosophiques sur les eléments de la science de l'étendue"). De hecho Gergonne fue el primero en utilizarla como principio en geometría.

${ }_{36}$ De l'analyse et de la synthèse dans les sciences mathématiques", p. 345. 
citas en general establece requisitos que sólo pueden cumplir algunos sistemas muy particulares - y ciertamente no el de Peano. Entonces, no se trata meramente de que Gergonne no aplique de hecho su noción de definición implicita a la base axiomática de los sistemas: no podía hacerlo. En ese sentido, los términos indefinidos, "definidos" mediante definiciones implícitas, tendrían serias limitaciones de usarse el significado de Gergonne, indudablemente más fuerte que el utilizado normalmente más tarde en sistemas axiomáticos. Como resultado podemos afirmar que las condiciones para el empleo de definiciones implícitas que derivan de su teoría general de la definición impidieron que Gergonne aplicara ese tipo de "definiciones" a la fundación de los sistemas.

4.5 Sin embargo, en Gergonne mismo encontramos las razones para el deslizamiento de significado que sufre la noción de definición implícita. La dualidad en la geometría proyectiva, con su intercambiabilidad de términos, preparó el camino para la consideración abstracta de los sistemas matemáticos. Si "punto" y "recta" - y también las relaciones entre ellos - son mutuamente sustituibles en la geometría proyectiva plana, entonces cada proposición adquiere un status potencialmente formal y cada uno de los términos pierde su sentido absoluto referente al mundo de las geometrías físicas. Eso es lo que Peano y especialmente Hilbert realizaron luego en sus campos respectivos -fundación de la aritmética y de la renovada geometría "euclideana" - permitiendo claramente la aplicación de las definiciones implícitas a los sistemas de axiomas. La ambigüedad de referencia de los términos definidos _-admitiendo un conjunto de modelos isomorfos- condujo al descrédito de la noción y la hizo inutilizable.

La construcción de un algoritmo consistente ${ }^{37}$ en la Dialectique Rationelle, en el cual el aspecto formal de la lógica apareció en vías hacia su plenitud, también dio otro paso en la misma dirección. Gergonne nos dice ${ }^{38}$ que, "heuresement", existen ciertos teoremas —en el sentido amplio de esta palabra - cuya verdad es evidente - los axiomas son justamente ellos-, pero luego nos mostró cómo los principios para desarrollar el algoritmo lógico no son absolutos y pueden intercambiarse. Tanto la dualidad en la geometría proyectiva como esta especial construcción lógica que constituye su algoritmo, con principios no fijados en permanencia, abrieron el camino para ulteriores desarrollos. Pero debemos recordar que las condiciones generales que Gergonne impuso a las definiciones le impidieron alcanzar la etapa hacia la cual apuntaban sus otros descubrimientos cientificos.

5. Traté de señalar las características que determinaron que la concepción de la lógica por parte de Gergonne diera un paso importante que los sucesores de Leibniz no lograran, y cómo Gergonne contribuyó a hacer obsoleto el dictum kantiano sobre la perfección y la imperfectibilidad de la lógica aristotélica.

37 Para un desarrollo de la Dialectique Rationelle de Gergonne y para una consideración de las propiedades formales de ese sistema, ver Faris, J. A., "The Gergonne Relations".

38 "De l'analyse et de la synthèse dans les sciences mathématiques", p. 345. 
El fundador de la nueva lógica - Boole - construyó verdaderamente un análisis matemático de la lógica; estudió, no "las leyes del pensamiento", sino las propiedades de algunos sistemas interpretados, y creó, no una escuela, sino una ciencia.

Gergonne no perpetuó su nombre, como Boole, en un conjunto de álgebras. Tuvo algunos vislumbres brillantes $y$, además, los tradujo en pasos concretos de construcción científica; pero, aun si contribuyó con ideas relevantes en varios dominios, no fundó la lógica de nuestro tiempo; por esta razón hemos tratado de mostrar cómo la noción de definición implícita por medio de axiomas _ sea en su estado más estricto o en su forma más popular y desacreditada - no fue ni podía ser uno de sus aportes. Traté de esbozar brevemente las razones de la importancia de Gergonne para el desarrollo de la lógica del siglo xIx. Lo que realmente cuenta en este sentido es el hecho de que sus contribuciones a la lógica, a la matemática, y a la filosofía, están integradas en la misma dirección de pensamiento. EI principio de dualidad, el desarrollo mismo de la geometría proyectiva sobre esa base, la construcción de un cálculo lógico puro (es decir, sobre el cual —dada su base- no actúen interpretaciones externas), sus teorías acerca del lenguaje cientifico y acerca de la definición - siendo ésta el motor de avance de la ciencia y aquél su característica más notoria-, son todos elementos unidos en una tendencia hacia la creación de una disciplina crecientemente formalizada, de un instrumento matemático y de una ciencia que utiliza las matemáticas: nuestra lógica.

InSTTtTUTO DE INVESTTGACIONES FILOSÓFICAS

Universidad Nacional Autónoma de México

Mario H. OTERo

\section{BIBLIOGRAFIA}

Bochenski, I., A History of Formal Logic, Notre Dame, University of Notre Dame, 1961.

Bowne, G., The Philosophy of Logic 1880-1908, The Hague, Mouton, 1966.

Carruccio, Mathematics and Logic in History and in Contemporary Thought, London, Faber \& Faber, 1964.

Castillon, G. F., "Reflexions sur la logique", Mémoires de l'Academie Royale des Sciences et Belles Lettres (Berlin), v. 52, 1804 (Classe de Philosophie Spéculative).

-_. "Mémoire sur un nouvel algorithme logique", Mémoires de l'Academie Royale des Sciences et Belles Lettres (Berlin), v. 53, 1805 (Classe de Philosophie Spéculative), pp. 3-24.

Cooley, J., A Primer of Formal Logic, New York, Macmillan, 1942.

Delboeuf, J., Essai de Logique scientifique, Liège, 1865.

-, "Logique algorithmique", Revue Philosophique de la France et de l'Etranger, 1876.

Enriques, F., The Historic Development of Logic, New York, Holt, 1929.

Faris, J., "The Gergonne Relations", The Journal of Symbolic Logic, v. 20, 1955, pp. 207-231. 
Gergonne, J.-D., "De l'analyse et de la synthèse dans les sciences mathématiques", Annales de Mathématiques Pures et Appliquées, v. 7, 1816-1817, pp. 345-372.

—, "Essai de dialectique rationelle", Annales..., v. 7, 1816-1817, pp. 189-228.

- "Essai sur la théorie des définitions", Annales..., v. 9, 1918-1919, pp. 1-35.

-, "Considérations philosophiques sur les éléments de la science de l'étendue", Annales de Mathématiques Pures et Appliquées, v. 16, 1826, pp. 209-232.

Kaminski, S., Gergonne'a teoria definicjii, Lublin, 1958.

Kneale, W. \& M., The Development of Logic, Oxford, Clarendon, 1962.

Lewis, C. I., A Survey of Symbolic Logic, Berkeley (California), 1918.

Otero, M., "Dos precursores de la lógica algorítmica: Castillon y Gergonne", Revista de la Facultad de Humanidades y Ciencias (Montevideo), 1963, pp. 5-19.

--, "Les definitions implicites chez Gergonne", Revue d'Histoire des Sciences et de leurs Applications (Paris), 1969.

-, (Abstract de) Mathematical Logic and its Philosophy in Early Nineteenth Cen. tury, XVth International Congress in the History of Science, Edinburgh, 1977. 